\title{
Relay Selection Scheme for Hierarchical Wireless Sensor Networks
}

\author{
Tran Nhon and Dong-Seong Kim \\ Department of IT Convergence, School of Electronic Engineering \\ Kumoh National Institute of Technology, South Korea \\ \{nhontran,dskim\}@kumoh.ac.kr
}

\begin{abstract}
This paper proposes a new relay selection scheme for wireless cooperative networks that effectively distributes the traffic of packets from the source nodes to the sink. By estimating the channel information of the two-hop neighbors, the source node can choose the most optimal relay node to forward its messages on the basis of the transmission rate of the channels from the source to the relay and from the relay to the destination. The simulation results indicate that our scheme is efficient in increasing the packet delivery ratio, decreasing the average energy consumption, and decreasing the end-to-end delay.
\end{abstract}

Keywords: Cooperative networks, relay selection, rate, packet delivery ratio, energy consumption, end-to-end delay

\section{Introduction}

Recently, wireless sensor networks (WSNs) have been identified as an attractive technology for industrial, factory automation, and distributed control systems [1, 2]. Wireless communication not only offers many advantages compared to wireline systems (e.g., users do not have to use a cord); however, it also suffers from many types of adverse effects such as path loss, thermal noise, and time variation. Moreover, one of the undesired effects of wireless communication systems is interference. It leads to severe reduction in the total throughput achieved in wireless networks.

It can be observed that one of the fundamental reasons for this poor performance is that reliable communication depends on the strength of the single signal path [3]. However, this path is regularly in a deep fade. When the path is in a deep fade, errors are likely to occur in any communication scheme. A basic solution to enhance the performance is to guarantee that the data can pass through multiple signal paths, which have independent fades. Therefore, communication is feasible as long as one of the paths is strong. This technique is called diversity, and it can enhance the performance over the fading channels.

There are three main types of diversity techniques, namely, time diversity, frequency diversity, and space diversity. Diversity can be achieved over time via coding and interleaving. Analogously, diversity over frequency can be exploited when the channel is frequency- selective, and one of the typical techniques of frequency diversity is orthogonal frequency-division multiplexing (OFDM). Diversity can be achieved over space as well in the cases where a channel with multiple transmit or receive antennas is spaced sufficiently. Multiple-input multiple-output (MIMO), one of the typical techniques of space diversity, is a promising technique for enhancing the performance in WSNs. It is a conventional form of spatial diversity with physical antenna arrays at both the transmitter and the receiver to 
improve communication. However, MIMO technology requires devices in the systems integrating several antennas. This is not feasible because of their size and weight limitations.

Cooperative communication (CC) has been proposed as a promising diversity technique to alleviate the burden of installing multiple antennas on size-limited terminals. By taking advantage of the broadcast nature of the wireless channels, multiple single- antenna terminals can collaboratively form a virtual antenna array (VAA) to exploit spatial diversity $[4,5]$. In wireless channels, transmission is overheard by the adjacent nodes. By taking advantage of this fact, a source node in CC can consider these adjacent nodes as relays and have them process and retransmit the signals to the destination. Further, the destination combines the signals received from the source and the relay, consequently achieving spatial diversity and robustness against the channel variations owing to fading. $\mathrm{CC}$ technology has been shown to significantly enhance the network performance in terms of saving power or energy $[6,7]$, reducing interference, and increasing throughput $[8,9]$. It can also allow the high-data-rate terminals to assist the low-data-rate terminals by relaying their data [10] such that higher transmission data rates can be achieved $[11,12]$. This is because CC can make robust the successful transmissions by offering duplicated signals at the receiver.

Typically, depending on the ability of the relay to decode the received signals from the source, there are two most regularly used strategies in $\mathrm{CC}$, which are the amplify-and-forward (AF) protocol [13] and the decode-and-forward (DF) protocol [14]. In the AF scheme, the relay receives and amplifies the incoming signals from the source, including noises. On the other hand, in the DF scheme, the relay decodes, re-encodes and transfers the messages from the source. At the destination, a suitable diversity- combining technique is used to recover the original messages from the source and relay. The authors in [15] mentioned that the AF protocol can be deployed in a wider area with a lower secrecy rate. By contrast, the deployment area of the DF protocol is narrower; however, it can get a higher secrecy rate. There are also some studies on the comparison between the performance of the AF and DF protocols $[16,17] . \mathrm{CC}$ is also categorized into two classes of solutions, namely reactive and proactive cooperative schemes [18]. In the former, the source node uses the cooperative scheme when it fails to transmit data to the destination. Conversely, with the latter, the source node always uses a neighboring node as the relay to transmit its packets to the destination on the basis of the knowledge of the channel state information (CSI).

One of the important tasks in $\mathrm{CC}$ is selecting the appropriate relays such that the performance of the network is optimized. There are some previous works on choosing the relay on the basis of the information on the paths. In [19], Bletsas et al. proposed a simple relay selection model to choose the best relay from a set of available neighbors. Nevertheless, their work considered only single-hop transmission with a fixed source-destination pair. Therefore, it is difficult to be extended to multi- hop/multi-source networks. In [20], Ibrahim et al. studied the energy-efficient cooperative routing algorithm by constructing a minimum power route named the minimum power cooperative routing (MPCR). This scheme, however, was mainly built to determine the optimal cooperative routes for the distributed shortest path algorithms. In [21, 22], the authors assumed that the rate is known; however, they did not describe how the source can obtain the rate information. In this paper, we propose a new method on the basis of the proactive cooperation scheme, by which the source node takes advantage of the advertising packet (ADV) periodically transmitted by all the nodes in the network to estimate the two-hop rates of the nodes around it. Consequentially, that source node can determine the appropriate neighbor node to become its relay. The simulation results show the efficiency of our scheme in increasing the packet delivery ratio, reducing the energy consumption, and decreasing the end-to-end delay. 
The remainder of this paper is organized as follows. We begin by describing the system model of our scheme in Section 2. Further, in Section 3, our method is explained in detail. The performance evaluation is discussed in Section 4. Finally, the conclusion and future work are presented in Section 5.

\section{System model}

\subsection{Channel model}

We assume that there are two nodes $i$ and $j$. When node $i$ transmits the data to node $j$, the signal that is received at node $j$ is described in equation 1 .

$$
y_{j}=h_{i j} \cdot x_{i}+n_{i j}
$$

where $x_{i}$ is the signal the source transmits to the destination and $y_{j}$ is the signal that the destination receives. $h_{i j}$ is the channel coefficient representing the effects of shadowing and fading between node $i$ and node $j$, and $n_{i j}$ is the additive white Gaussian noise (AWGN). On the basis of the received signal, node $j$ calculates the rate of the path from node $\mathrm{i}$ to node $\mathrm{j}$ by equation 2 .

$$
R_{i j}=W \cdot \log _{2}\left(1+\frac{P \gamma_{i j}}{N_{0} d_{i j}^{\alpha}}\right)
$$

where $W$ is the channel bandwidth; $P$ is the transmitter power; $N_{0}$ is the AWGN; $d_{i j}$ is the distance between nodes $i$ and $j ; \alpha$ is the path-loss exponent, which is between 2 and 4 in general; and $\gamma_{i j}=\left|h_{i j}\right|^{2}$.

\subsection{Cooperative communication model}

In this paper, we consider a cooperative scheme with a simple system that includes one source, one relay, and one destination, each with a single antenna, which is shown in Figure 1.

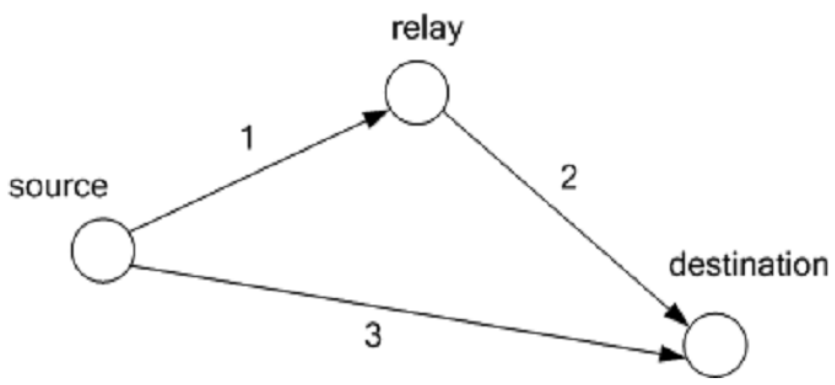

Figure 1. Typical cooperative network

The $\mathrm{CC}$ process in our scheme includes two phases. In the first phase, the message is transmitted by the source to the relay and the destination. In the second phase, the source and the relay transmit the message to the destination. Therein, we apply the IEEE 802.11 CoopMAC protocol, as in [8, 23]. In CoopMAC, there are some modifications based on the original MAC protocol. First, the address of the relay node 
also exists in the request-to-send (RTS) frame. Second, there is a special packet, called clear-to-relay (CTR) packet, transmitted by the relay to acknowledge that it is ready to relay. The operation of the source, relay, and destination nodes is described as follows:

- Source node $S$ : Whenever there is at least one packet buffered in the queue, it broadcasts the RTS packet to the nodes around it. After receiving the clear-to-send (CTS) and CTR packets from the destination and relay, the source transmits the data packet in two phases (or two time slots), as explained above.

- Relay node $R$ : After receiving the RTS from the source and CTS from the destination, the relay broadcasts the CTR to the source and the destination to inform that it is ready to aid the source to transmit the data to the destination. Further, the message from the source arrives at the relay in the first phase. In the second phase, the relay transmits that message to the destination.

- Destination node D: When the destination receives an RTS from the source, it then replies by trasmitting the CTS packet back to the source and the relay. When the message from the source is received after the CTR packet from the relay, the destination waits for another message in the following phase.

On the basis of the above explanations the received signal at the relay - $y_{s r}$ and that at the destination from the source/relay $-y_{s d}$, and $y_{r d}$ are written as in following equations:

$$
\begin{aligned}
& y_{j}=h_{i j} \cdot x_{i}+n_{i j} \\
& y_{j}=h_{i j} \cdot x_{i}+n_{i j} \\
& y_{j}=h_{i j} \cdot x_{i}+n_{i j}
\end{aligned}
$$

where $x_{s}$ is the transmitting signal at the source, whereas $x_{r}$ is the signal that is detected and forwarded to the destination by the relay. $h_{s r}, h_{s d}$, and $h_{r d}$ are the channel coefficients representing the effects of shadowing and fading between the source and the relay, between the source and the destination, and between the relay and the destination respectively. $n_{s r}, n_{s d}$, and $n_{r d}$ are the additive noise components in the received signals at the respective paths.

The analyses of three types of linear-diversity-combining techniques at the destination are mentioned in [24], including selection diversity, maximal-ratio diversity, and equal-gain diversity systems. It is observed that the diversity techniques mitigate the effects of fading and improve the link quality of the wireless channel. Our scheme adopts the concepts of diversity-combining methods applied in the analog domain at the physical layer to the received signals at packet level relying on the CSI.

This work is inspired by multi-hop wireless networks with a proactive traffic-aware routing protocol (TADR) [25]. The nodes in the network update the network information and decide on the destination node from the surrounding nodes. Ren et. al [25] proposed a routing scheme that used the concept of the potential in physical to use the idle or underloaded nodes in the forwarding path to reduce congestion and improve the overall throughput in WSNs efficiently. By combining CC and the background routing algorithm, we propose a new reliable scheme on the basis of rate-aware mechanism to enhance the network performance. The main objective of our work is to determine the reliable relay for each source-destination pair. The relay selection exploits the instantaneous channel measurement to decide the winner to become the 
relay, that is, our selection mechanism chooses the strongest link among the relays to both the source and the destination which can transfer the message from the source as well.

\subsection{Relay selection}

To model the relay selection algorithm, we investigate a communication system that includes one source, one destination, and $k$ candidates for the relay. In this case, the destination and the candidates are in the transmission range of the source. This system uses the proactive protocol such that the source always builds a table that includes the information on the rate of all the possible candidates in the network.

On the basis of the information in the table that the source node built, the $A$ value is calculated following equation 6 .

$$
A=\frac{1}{R_{r s}}+\frac{1}{R_{r d}}-\frac{1}{R_{s d}}
$$

where $R_{s r}, R_{r d}$, and $R_{s d}$ represent the storage of the data rate from the source to the relay, from the relay to the destination, and from the source to the destination, respectively. For each relay, when $A>0$, it implies that it takes a longer time to transmit the data through the path using that relay than the direct path. In contrast, when $A<0$, it implies that it obtains benefits when transmitting the data toward the path using that relay, which can be understood that the relays that are chosen are located close to the transmitter or receiver [8]. The probability of this occurring can be written as follows:

$$
P_{r}=P(\mathrm{~A}<0)
$$

As in this heading, they should be Times New Roman 11-point boldface, initially capitalized, flush left, with one blank line before, and one after.

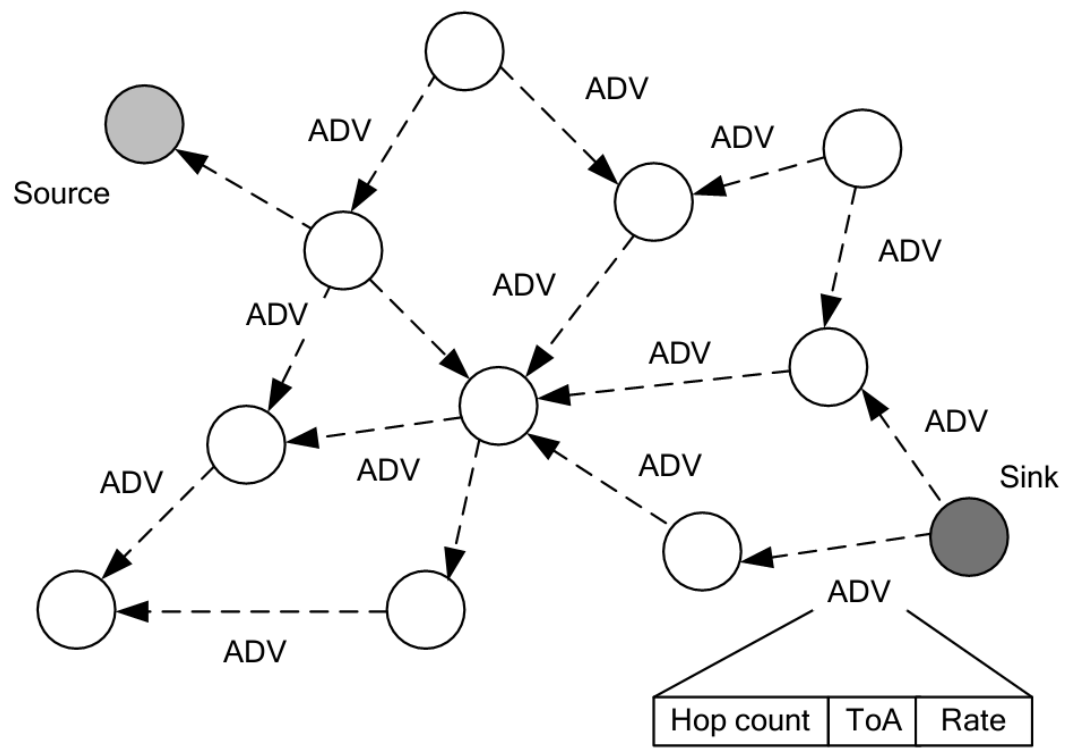

Figure 2. Update table based on the ADV packets 


\subsection{Network topology}

In a network, when the source has to transmit data to the sink, it always chooses the shortest path to the sink to send data. For this, all the nodes including the sink periodically broadcast the ADV, which includes information on the hop count, time of arrival (ToA), and rate of the paths to all the other nodes in the network, as shown in Figure 2. When the ADV arrives in the source, the source is able to determine the path that has the lowest hop count from it to the sink.

\subsection{Network topology}

The average outage probability is also one of the important performance metrics of a wireless cooperative network [26]. In any analysis, there is always the assumption that the channel SNR is measured reliably. An outage event is the case when reliable decoding for a fixed rate cannot occur when the instantaneous SNR drops below a certain threshold. By assuming that there is a set of forwarding relays in a network, $R=$ $r_{1}, \ldots, r_{L}$, which have transmitted without collisions. Further, the destination $d$ combines the signals received from $L$ relays and obtains the combined SNR as follows:

$$
\gamma_{c}=\gamma_{0}+\sum_{l=1}^{L} \gamma_{l}
$$

Where $\gamma_{0}$ and $\gamma_{l}$ denote the received SNR for $s-d$ and $r_{l}-d$ channels, respectively. When $\gamma_{\mathrm{c}}$ is less than the threshold $T$ for guaranteeing successful decoding at $d$, an outage occurs with a probability $P_{\text {out }}=\operatorname{Pr}\left[\gamma_{c}<T\right]$. Relay decisions can be made on the basis of the single-threshold test [27, 28] and double-threshold test [29, 30]. In [26], Kuang et al. proposed a fully distributed threshold relaying scheme, called distributed threshold relaying (DTR) to analyze the performance of threshold relaying with random channel access over non-identically distributed Rayleigh-fading channels. The problem of how to reduce the outage probability is one of the most important tasks in improving the performance of wireless networks. However, this problem will be addressed the future works.

\section{Proposed Methods}

\subsection{Destination selection}

First, each node, including the sinks, periodically broadcasts an ADV packet to all the neighbors after a predefined period of time. On the basis of this process, each node maintains a table of potential destinations and relays that can be used to transmit its data.

In the algorithm 1 , to choose the destination, when the source node has data of $\mathrm{L}$ octets to transmit, it monitors each entry of the table to decide whether to transmit through a particular node. First, the source compares the hop count of the neighbors in its transmission range. With the neighbors that have a hop count lower than that of the source, it adds them to the list of its parents. However, with the neighbors that have an equal hop count; it adds them to the list of its siblings. Furthermore, the source node does not use the neighbors that have a hop count bigger than it. 


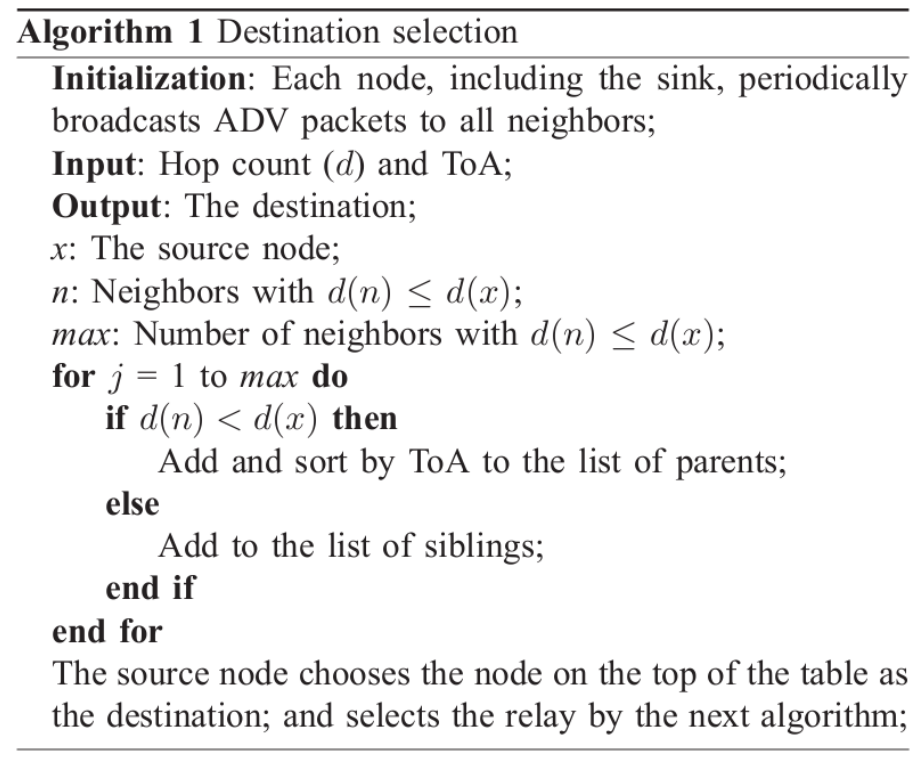

After building a table that consists of parents and siblings, from the list of parents, the source node chooses the destination as the node that has the smallest ToA. This implies that the parent that is the nearest from the source.

\subsection{Relay selection}

Algorithm 2 describes the relay selection scheme. After the destination is selected, the remaining nodes in the table are candidates to become the source's relays. In those nodes, the source calculates the $A$ values through the two-hop rate information of each one. When all of them have $A>0$, the source transmits its data directly to the destination. In this case, it is not effective to transmit data via the relay. However, when some of them have $A<0$, the source chooses the node that has the lowest value of $\mathrm{A}$ as the relay.

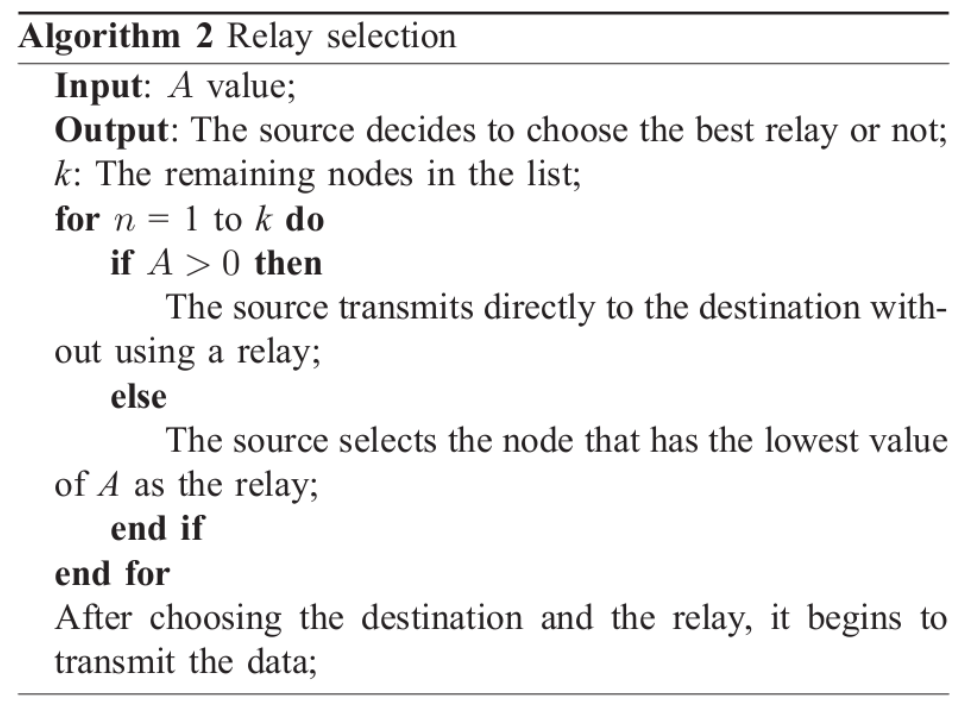




\section{Simulation and Evaluation}

\subsection{Simulation setup}

The proposed schemes are evaluated by OPNET modeler 16.0. The network includes 100 nodes, which are randomly distributed in a $200 \mathrm{~m} \times 200 \mathrm{~m}$ square area. In our simulations, we set a bandwidth of $W=22 \mathrm{MHz}$ for each channel. For each node, the number of incoming data packets is exponentially distributed with a given $\lambda$. We implement the 802.11 MAC protocol in the proposed scheme. The IEEE MAC performs an exponential backoff when a node is involved in a collision. In particular, when there is a collision, each node picks a random number $X$ from $[0, C W]$, where $C W$ is the current size of the congestion window, and it retransmits after $X$ time slots. When the retransmission is unsuccessful, $C W=\min (2 \times C W$, $C W \max$ ), otherwise $C W$ is set to $C W m i n$. The expected time spent in the contention procedure for each node increases with the number of nodes in the network. In our work, we assume that the network is heavily loaded. This implies that there is always at least one packet in the buffer that awaiting to transmit at each node. The simulation result is obtained by averaging over 10 runs of simulations. The detailed simulation parameters are listed in Table 1.

Table 1. Simulation Parameters

\begin{tabular}{|l|l|}
\hline Parameters & Values \\
\hline Deployment type & Random topology \\
Deployment area & $200 \times 200 \mathrm{~m}^{2}$ \\
Number of nodes & 100 \\
MAC protocol & CSMA/CA for 802.11 \\
$C W_{\min }$ & 32 \\
$C W_{\max }$ & 1024 \\
Slot time & $20 \mu \mathrm{s}$ \\
Radio power & $0.05 \mathrm{~mW}$ \\
Carrier frequency & $2.4 \mathrm{GHz}$ \\
Packet size & $1000 \mathrm{~B}$ \\
Data rate & $11 \mathrm{Mbps}$ \\
Simulation time & $1000 \mathrm{~s}$ \\
Traffic type & Exponential distribution \\
\hline
\end{tabular}

\subsection{Numerical results}

The simulation results are subsequently compared with the transmission without using any relay, i.e, with direct channels only, a traffic-balancing routing scheme (TBRS) [31], and a cooperative scheme based on the rate of the first hop from the source to the relay to show the effectiveness of the proposed scheme in increasing the packet delivery ratio and decreasing the average energy consumption.

4.2.1. Average energy consumption: Figure 3 shows the average energy consumption per bit for each received packet at the sink. It can be observed that the average energy per bit in the case of the proposed scheme (two-hop rate-aware) considerably less than that of direct transmission, that is, $28.5 \%$. Moreover, it is $11 \%$ less than that of the TBRS and first hop rateaware. This is because of the fact that without considering the channel condition of the path, the transmission suffers from high collision; therefore, the number of retransmissions is high, and the network consumes high energy. Furthermore, in some cases, there is no need to use a 
relay when the path through the relay is not effective as the direct path. Hence, the two-hop rate-aware scheme can aid the network to save energy.

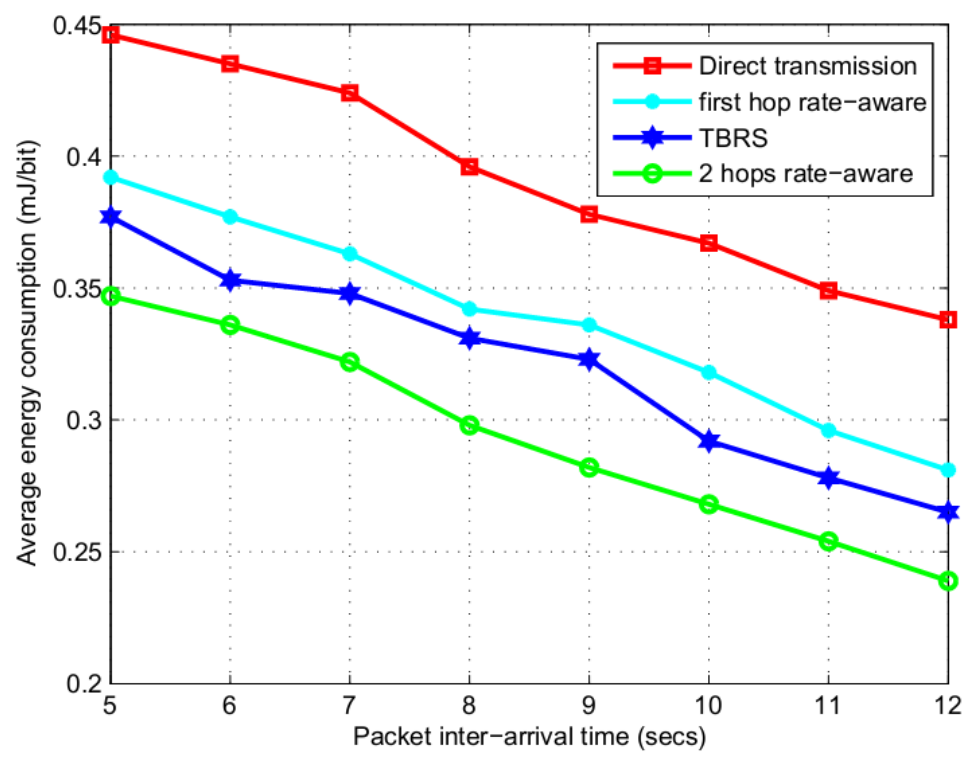

Figure 3. Average energy consumption per bit successfully received at the sink versus different values of inter-arrival time

4.2.2. Packet delivery ratio: We have evaluated the effects of the proposed scheme on the packet delivery ratio. Traffic is generated following the exponential distribution. When the packet inter-arrival time is small, higher traffic is generated from the source nodes. This increases packet collision, leading to a lower packet delivery ratio. Figure 4 shows that the proposed method improves the packet delivery ratio from $9 \%$ to $13 \%$ when compared with direct transmission without using a relay and from $4.1 \%$ to $5.7 \%$ when compared with the TBRS and another relay selection scheme that is based on a single hop from the source to the relay. The destination has two chances to receive the messages when applying the cooperative scheme. Furthermore, the sources can choose the optimized relays, corresponding to the optimized paths to transmit their messages through rate information in two hops from the source to the relay and from the relay to the destination. Hence, it can achieve a higher packet delivery ratio compared with the single hop from the source to the relay rate information, because the channel from the relay to the destination could be in very bad condition such that the entire path using that relay is not the most optimal one.

4.2.3. Average end-to-end delay: Figure 5 shows the average end-to-end delay of the three schemes. The tendency of the end- to-end delay is similar to that of the packet delivery ratio. It can be observed that the average end-to-end delay when applying the proposed method is always lower than that of direct transmission, TBRS, and first hop-rate-aware. Furthermore, direct transmission achieves the worst results among the three schemes. With direct transmission, the nodes transmit packets with minimum hops; however, this transmission is through low-quality channels. Consequently, the packet loss at the destination increases. There are, therefore, many packets that require to be retransmitted. Whereas in contrast, with the other schemes, the packets that are based on the channel estimation are transmitted with higher reliability, leading to lower retransmissions, particularly in the case of the proposed scheme. As a result, the packets reach the sink with a lower delay. 


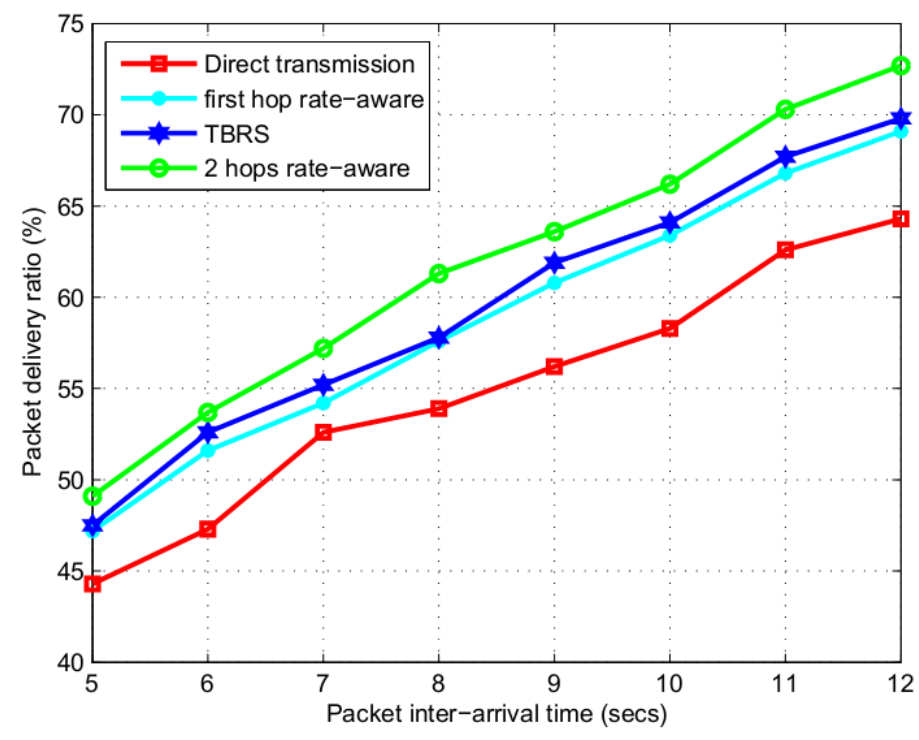

Figure 4. Packet delivery ratio versus different values of inter-arrival time

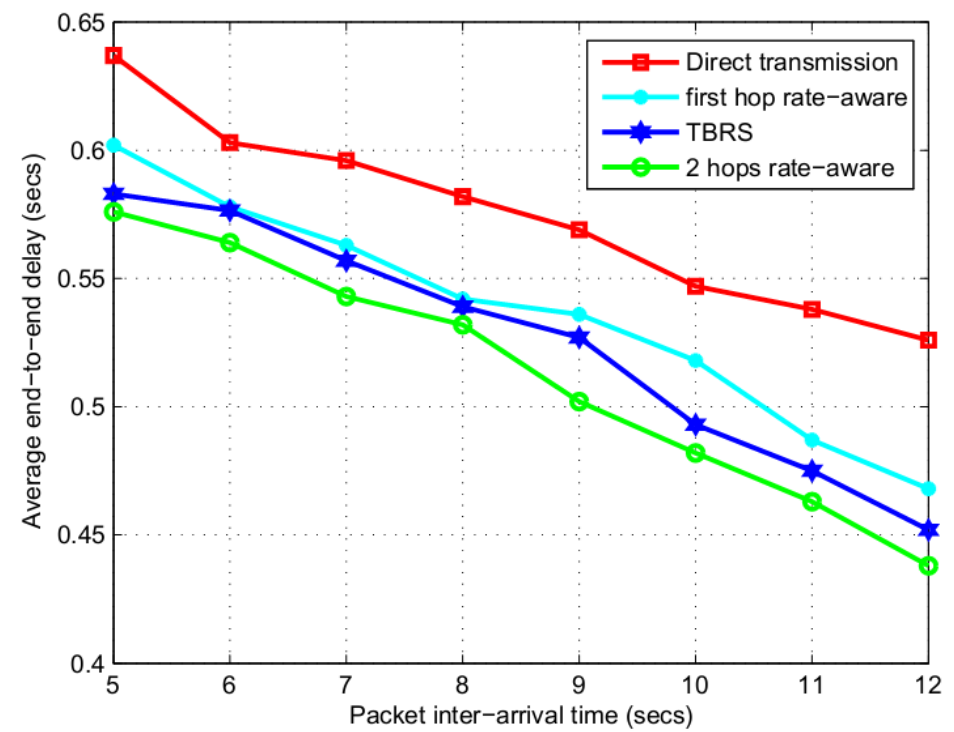

Figure 5. Average end-to-end delay versus different values of inter-arrival time

\section{Conclusion}

In this paper, we proposed a reliable rate-aware relay selection method, where the source exploits the CSI of the paths from the source to relay and from the relay to the destination as well to choose the most reliable node to become the relay. The numerical and simulation results confirmed that the proposed scheme significantly outperforms the direct transmission and the method based on the rate information in the first hop. To address the practical concerns, the proposed algorithm can be easily implemented on existing devices without major changes. 
As a future direction on this research, we plan to investigate the algorithm to overcome the negative effects of time-variant; we also consider efficient power allocation and applying the beamforming scheme to our scheme.

\section{Acknowledgements}

This research was financially supported by the Ministry of Education, Science Technology (MEST) and National Research Foundation of Korea (NRF) through the Human Resource Training Project for Regional Innovation 2013.

\section{References}

[1] D. Choi and D. -S. Kim, "Wireless Fieldbus for Networked Control Systems using LR-WPAN", International Journal of Control and Automation and Systems, vol. 6, no. 1, (2008) February, pp. 119-125.

[2] D. D. Tan, N. Q. Dinh and D. -S. Kim, "GRATA: A gradient-based traffic-aware routing for wireless sensor networks", IET Wireless Sensor Systems, vol. 3, no. 2, (2013) June, pp. 14 -20.

[3] P. T. A. Quang and D. -S. Kim, "Throughput-aware Routing for Industrial Sensor Networks: Application to ISA100.11a", IEEE Transactions on Industrial Informatics, vol. 10, no. 1, (2014) February, pp. 351-363.

[4] B. Zhao and M. Valenti, "Practical relay networks: a generalization of hybrid-ARQ", IEEE Journal on Selected Areas in Communications, vol. 23, no. 1, (2005), pp. 7-18.

[5] H. Shan, H. T. Cheng and W. Zhuang, "Cross-Layer Cooperative MAC Protocol in Distributed Wireless Networks", IEEE Transactions on Wireless Communications, vol. 10, no. 8, (2011), pp. 2603-2615.

[6] J. Laneman, D. Tse and G. W. Wornell, "Cooperative diversity in wireless networks: Efficient protocols and outage behavior", IEEE Transactions on Information Theory, vol. 50, no. 12, (2004), pp. 3062-3080.

[7] J. Laneman and G. Wornell, "Energy-efficient antenna sharing and relaying for wireless networks", in IEEE Wireless Communications and Networking Confernce, vol. 1, (2000), pp. 7-12.

[8] P. Liu, Z. Tao, S. Narayanan, T. Korakis and S. Panwar, "CoopMAC: A Cooperative MAC for Wireless LANs", IEEE Journal on Selected Areas in Communications, vol. 25, no. 2, (2007), pp. 340-354.

[9] Z. Sheng, K. Leung and Z. Ding, "Cooperative wireless networks: from radio to network protocol designs", IEEE Communications Magazine, vol. 49, no. 5, (2011), pp. 64-69.

[10] N. Tadayon, H. Wang, D. Kasilingam and L. Xing, "Analytical Modeling of Medium-Access Delay for Cooperative Wireless Networks Over Rayleigh Fading Channels", IEEE Transactions on Vehicular Technology, vol. 62, no. 1, (2013), pp. 349-359.

[11] A. Sendonaris, E. Erkip and B. Aazhang, "User cooperation diversity. Part I. System description", IEEE Transactions on Communications, vol. 51, no. 11, (2003), pp. 1927-1938.

[12] "User cooperation diversity. Part II, Implementation aspects and performance analysis", IEEE Transactions on Communications, vol. 51, no. 11(2003), pp. 1939-1948.

[13] H. Bolcskei, R. Nabar, O. Oyman and A. Paulraj, "Capacity scaling laws in MIMO relay networks", IEEE Transactions on Wireless Communications, vol. 5, no. 6, (2006), pp. 1433-1444.

[14] C. Gong, A. Tajer and X. Wang, "Group Decoding for Multi-Relay Assisted Interference Channels", IEEE Journal on Selected Areas in Communications, vol. 30, no. 8, (2012), pp. 1489-1499.

[15] P. Zhang, J. Yuan, J. Chen, J. Wang and J. Yang, "Analyzing Amplify-and-Forward and Decode-andForward Cooperative Strategies in Wyner's Channel Model", in IEEE Conference on Wireless Communications and Networking, (2009), pp. 1-5.

[16] M. Yu and J. Li, "Is amplify-and-forward practically better than decode-and-forward or vice versa?", in IEEE International Conference on Acoustics, Speech, and Signal Processing, vol. 3, (2005), pp. 365-368.

[17] M. Souryal and B. Vojcic, "Performance of Amplify-and-Forward and Decode-and-Forward Relaying in Rayleigh Fading with Turbo Codes", in IEEE International Conference on Acoustics, Speech and Signal Processing, vol. 4, (2006).

[18] A. Munari, M. Levorato and M. Zorzi, "On the Impact of Carrier Sense Based Medium Access Control on Cooperative Schemes in Wireless Ad Hoc Networks", IEEE Transactions on Communications, vol. 60, no. 10, (2012), pp. 3032-3046. 
[19] A. Bletsas, A. Khisti, D. Reed and A. Lippman, "A simple Cooperative diversity method based on network path selection", IEEE Journal on Selected Areas in Communications, vol. 24, no. 3, (2006), pp. 659-672.

[20] A. Ibrahim, Z. Han and K. Liu, "Distributed energy-efficient cooperative routing in wireless networks", IEEE Transactions on Wireless Communications, vol. 7, no. 10, (2008), pp. 3930-3941.

[21] T. Pham, H. Nguyen and H. Tuan, "Relay Assignment for Max-Min Capacity in Cooperative Wireless Networks", IEEE Transactions on Vehicular Technology, vol. 61, no. 5, (2012), pp. 23872394.

[22] P. T. A. Quang and D. -S. Kim, "Enhancing Real-Time Delivery of Gradient Routing for Industrial Wireless Sensor Networks", IEEE Transactions on Industrial Informatics, vol. 8, no. 1, (2012) February, pp. 61-68.

[23] M. Gokturk and O. Gurbuz, "Cooperation in Wireless Sensor Networks: Design and Performance Analysis of a MAC Protocol", in IEEE International Conference on Communications, (2008), pp. 4284-4289.

[24] D. Brennan, "Linear deversity combining techniques", in Proceedings of the IEEE, vol. 91, no. 2, (2003) February, pp. 331-356.

[25] F. Ren, T. He, S. Das and C. Lin, "Traffic-Aware Dynamic Routing to Alleviate Congestion in Wireless Sensor Networks", IEEE Transactions on Parallel and Distributed Systems, vol. 22, no. 9, (2011), pp. 1585-1599.

[26] K. -H. Liu and H. -H. Chen, "Performance Analysis of Threshold Relaying with Random Channel Access over Non-Identically Distributed Rayleigh-Fading Channels", IEEE Journal on Selected Areas in Communications, vol. 30, no. 9, (2012), pp. 1703-1710.

[27] W. Siriwongpairat, T. Himsoon, W. Su and K. Liu, "Optimum threshold-selection relaying for decode-and-forward cooperation protocol", in IEEE Wireless Communications and Networking Conference, vol. 2, (2006), pp. 1015-1020.

[28] A. Adinoyi and H. Yanikomeroglu, "Cooperative relaying in multi-antenna fixed relay networks", IEEE Transactions on Wireless Communications, vol. 6, no. 2, (2007), pp. 533-544.

[29] K. -H. Liu, "Double Threshold Digital Relaying for Cooperative Wireless Networks", in IEEE Vehicular Technology Conference, (2010), pp. 1-5.

[30] Y. Li and Q. Zhang, "Distributed Relay Selection Scheme in Decode-and-Forward Cooperative Systems", in IEEE 71st Vehicular Technology Conference, (2010), pp. 1-5.

[31] D. D. Tan and D. -S. Kim, "Dynamic traffic-aware routing algorithm for multi-sink wireless sensor", Wireless Networks (Springer), (2014).

\section{Authors}

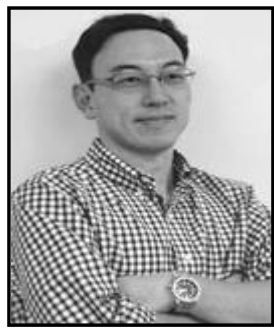

Dong-Seong Kim received his Ph.D. degree in Electrical and Computer Engineering from the Seoul National University, Seoul, Korea, in 2003. From 1994 to 2003, he worked as a full-time researcher in ERC-ACI at Seoul National University, Seoul, Korea. From March 2003 to February 2005, he worked as a postdoctoral researcher and visiting scholar at the Wireless Network Laboratory in the School of Electrical and Computer Engineering at Cornell University, NY. He was a visiting professor with department of computer science, University of California, Davis, U.S.A. Since 2004, he has been a professor in the School of Electronic Engineering and chair of mobile research center at Kumoh National Institute of Technology, Korea. His current main research interests are industrial wireless control network, networked embedded system and Fieldbus. 


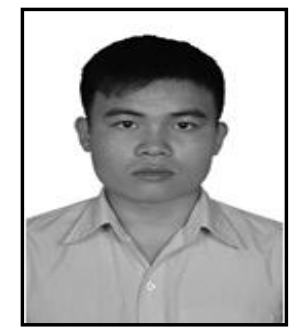

Tran Nhon is currently a Master student and researcher at Networked Systems Lab, Kumoh National Institute of Technology. He received the Bachelor's degree from Ho Chi Minh City University of Industry in 2012. His main areas research interests are wireless networks and realtime systems, cooperative communication. 
International Journal of Control and Automation Vol.7, No.3 (2014) 